\title{
Task selection cost asymmetry without task switching
}

\author{
RICHARD L. BRYCK AND ULRICH MAYR \\ University of Oregon, Eugene, Oregon
}

\begin{abstract}
The switch cost asymmetry (i.e., larger costs when switching from a nondominant into a dominant task than vice versa) has been explained in terms of the trial-to-trial carryover of activation levels required for the dominant versus the nondominant task. However, there is an open question about whether an actual switch in task is in fact necessary to obtain a "selection" cost asymmetry. In Experiments 1 and 2, we modified an alternatingruns paradigm to include either long or short response-to-stimulus intervals (RSIs) after each pair of trials (i.e., AA-AA-BB-BB), thereby inducing selection costs not only at the point of a task switch (i.e., AA-BB), but also between same-task pairs (i.e., AA-AA). Using spatially compatible versus incompatible response rules (Experiment 1) and Stroop word versus color naming (Experiment 2), we found asymmetric effects not only at task-change transitions, but also at task-repeat transitions when the RSI was long (presumably inducing frequent losses of set). In Experiments 3A and 3B, a cost asymmetry for long RSIs was obtained even when competing tasks were separated into alternating single task blocks, but not when the tasks were compared in a betweensubjects design. This general pattern cannot be explained by activation carryover models, but is consistent with the idea that the asymmetry arises as a result of interference from long-term memory traces.
\end{abstract}

How people perform in multitask situations can provide important information about the architecture of cognitive control. One particularly intriguing result is the so-called switch cost asymmetry. This phenomenon, first described by Allport, Styles, and Hsieh (1994), occurs when participants switch back and forth between two tasks that differ in relative dominance, such as Stroop word naming versus color naming. In such situations, switching from the less dominant to the more dominant task takes longer than vice versa. In other words, it seems more difficult to establish an easy task than to establish a difficult task. The paradoxical nature of this effect makes it an important challenge for models of executive control.

So far, the dominant account of both switch costs and the switch cost asymmetry is in terms of trial-to-trial carryover of relative levels of activation for the dominant versus the nondominant task within a connectionist-type model (e.g., Gilbert \& Shallice, 2002; Yeung \& Monsell, 2003). We refer to this class of models as carryover accounts of task switching. For example, in Gilbert and Shallice's computational model, selection between two tasks occurs through the relative activation of two "task demand nodes" that exert top-down control on lower level, task-specific nodes. Dominant task pathways (e.g., Stroop word reading) are characterized by stronger associations than nondominant task pathways (e.g., Stroop color naming) are. Thus, to select the dominant task, little additional activation of the corresponding task node and little suppression of the nondominant task node are needed. Ac- cording to Gilbert and Shallice, the cost asymmetry then arises because

a larger value is added to the net input of the color task demand unit on color trials than is added to the net input of the word task demand unit on word trials. Thus, on switch trials, reconfiguration of the task demand units for the intended task is slower for the word-reading task than [for] the color-naming task, hence the paradoxical asymmetry in switch costs.

(p. 317) 1

The carryover account is parsimonious and intuitively appealing. It basically reduces the problem of switching between tasks to that of a network's requiring more time (on switch trials) or less time (on no-switch trials) before settling into a state that allows successful response selection. In fact, Gilbert and Shallice (2002) suggested that, given the success of their model, assumptions about "extra" processes that might be relevant for task selection, but not for response selection, are unnecessary.

However, carryover accounts also make a straightforward prediction: Given that the reason for the cost asymmetry lies in the carryover of activation between consecutive trials, task-switch transitions are a necessary condition for obtaining a cost asymmetry. In other words, carryover accounts could not explain cost asymmetries that occur in the absence of a switch in task.

Cost asymmetries in the absence of switch transitions can also be explained by a class of models that differenti-

R. L. Bryck, rbryck@uoregon.edu 
ate between two distinct processes: (1) task maintenance in working memory, across no-switch transitions, and (2) retrieval of task rules from long-term memory (LTM) usually limited to switch transitions (e.g., Allport \& Wylie, 2000; Hazy, Frank, \& O’Reilly, 2006; Mayr \& Kliegl, 2000, 2003). According to such LTM-retrieval views of selection, asymmetric costs could arise from interference of LTM traces of previous selection instances whenever a task-set needs to be retrieved from LTM, even when this does not occur in the context of a task-switch transition. In the Discussion, we will further elaborate on how the cost asymmetry can arise as a result of LTM interference. At this point, the critical aspect is that, by the LTM view, switch transitions would be a sufficient, but not necessary, condition for obtaining a cost asymmetry.

Interestingly, it has been known for some time that substantial performance costs arise not only at switch transitions, but also when ongoing performance is interrupted by a short pause (Gopher, Armony, \& Greenshpan, 2000). Furthermore, according to a hitherto largely ignored finding by Allport and Wylie (2000), such "restart" costs show asymmetries similar to actual switch costs. The authors explicitly interpreted this result as being inconsistent with the idea that "persisting facilitation or suppression of competing processing pathways" (p. 49) is responsible for switch costs and the cost asymmetry.

The goal of the present study was to replicate (Experiments 1 and 2) and expand (Experiments 3A and 3B) Allport and Wylie's (2000) findings. In Experiments 1 and 2, we used a variant of the alternating-runs paradigm (Rogers $\&$ Monsell, 1995). We adapted this paradigm to create situations in which participants were likely to re-retrieve task sets from LTM, even when the task did not change from the previous trial (Figure 1; see Method for detailed explanation).

Specifically, we speculated that with long response-tostimulus intervals (RSIs), there should be a greater probability of losing the current task set from working memory than with short RSIs. Thus, we predicted a selection cost asymmetry not only after task-switch transitions, but also after long-RSI, no-switch transitions. Note that we expected that a long RSI would increase the probability that re-retrieval of task sets would be required on taskrepetition trials, but this does not imply that retrieval never occurs after short RSIs. Thus, the critical prediction is that of a cost asymmetry for the task-repetition transitions, but it is less important that the cost asymmetry be reliably modulated by the short/long RSI factor. In Experiments 1 and 2, we used two different sets of tasks with established dominance relationships: stimulus-response-compatible versus stimulus-response-incompatible mappings (i.e., S-R compatibility) and Stroop word naming versus Stroop color naming (i.e., Stroop task).

\section{EXPERIMENTS 1 AND 2}

\section{Method}

Participants. Thirty-six University of Oregon students participated in Experiment 1 (S-R compatibility) in exchange for course credit or payment of US\$7, and 30 students participated in Experiment 2 (Stroop).

\section{Compatible}

Task
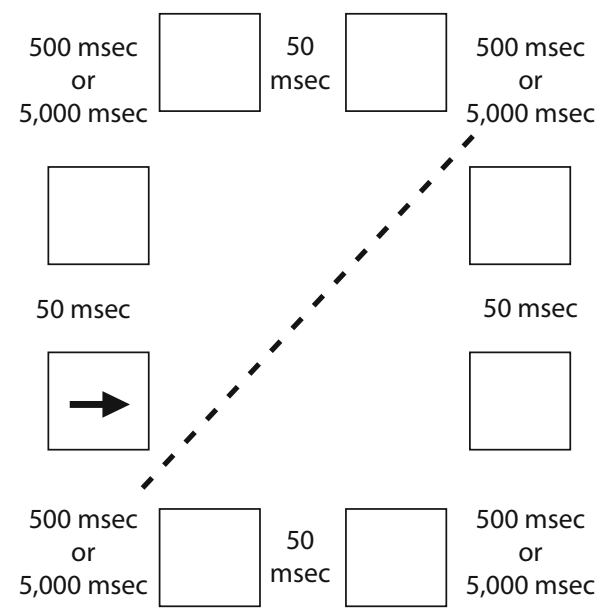

$500 \mathrm{msec}$$$
\text { or }
$$
$5,000 \mathrm{msec}$

Incompatible Task

Figure 1. Schematic representing the task design for Experiment 1 . Stimuli were presented one at a time in one of the eight boxes, rotating clockwise through the $\mathrm{AA}-\mathrm{AA}-\mathrm{BB}-\mathrm{BB}$ task sequence. Tasks were defined spatially, with the compatible task to be performed in the upper-left half of the display and the incompatible task in the lower-right half of the display; the dashed line represents the task boundary. Within-pairs RSIs were short $(50 \mathrm{msec})$, while RSIs between pairs of trials varied between short $(500 \mathrm{msec})$ and long $(5,000 \mathrm{msec})$. These between-pairs RSIs (shown at the corners in the figure) allowed for the different task transition types (task change and task repetition). The actual display shown to participants only included the task stimuli for a given trial and the eight boxes (i.e., it did not include the task cues, dashed line, or RSI label). The design was identical for Experiment 2 except that within-pairs RSIs were $500 \mathrm{msec}$ (to accommodate for the vocal response method), and the stimuli presented in each box were Stroop color words, with the two tasks being word naming and color naming.

General Design. We used an alternating-runs paradigm in which participants selected between two different tasks in two runs of four trials (i.e., AAAABBBB). See Figure 1 for a schematic representation of how tasks were presented in Experiment 1. (Note that only the boxes and the stimuli were actually shown to participants; task presentation in Experiment 2 was analogous.) Stimuli were presented one at a time, rotating clockwise through the eight boxes displayed on the screen sequentially. The same-task runs were further broken down into pairs of two same-task trials (i.e., AA-AA-BB-BB). This design allowed for two types of between-pairs transitions: task-change transitions (i.e., $\mathrm{AA}-\mathrm{BB}$ and $\mathrm{BB}-\mathrm{AA}$ ) and task-repetition transitions (i.e., AA-AA and BB-BB). The RSIs between two within-pairs trials was very short $(50 \mathrm{msec})$ and held constant. However, the RSIs between pairs of trials varied randomly between short $(500 \mathrm{msec})$ and long $(5,000 \mathrm{msec})$. With this design, we capitalized on the fact that participants have a strong tendency to group sequences of tasks into smaller chunks (e.g., Lien \& Ruthruff, 2004).

Therefore, we expected that participants would often need to reretrieve the current task from LTM, even when the relevant task did not change. The measurement of "selection cost" we used was the difference between the first and the second position within a pair, irrespective of transition type. We expected this need to re-retrieve the task to be particularly true for the long $(5,000 \mathrm{msec})$ RSI, given the higher probability of losing the relevant set from working memory after longer delays. 
Stimuli, Tasks, and Procedure. Stimuli in Experiment 1 were two arrows: one pointing to the left and one pointing to the right. Responses were made with the left and right arrow keys of the keyboard. In the dominant task condition, participants responded in a compatible manner (i.e., left key for left arrows), and in the nondominant task condition, participants responded in an incompatible manner (i.e., left key for right arrows). Participants began with a block of 96 practice trials, alternating between the dominant and nondominant tasks as described above. Participants then completed nine more blocks, for a total of 864 trials (excluding practice).

Stimuli in Experiment 2 were the words red, green, blue, and yellow displayed in the colors red, green, blue, or yellow. Only incongruent stimuli were used (i.e., the color and the name of the word were always inconsistent), resulting in 12 unique stimuli. Participants made vocal responses to the stimuli based on the experimental condition-dominant responses required producing the name of the word (word naming), while nondominant responses required saying the color of the word (color naming). An experimenter was present to record errors and monitor performance. The experiment began with two 48-trial blocks of single-task practice, with the order of task presentation counterbalanced across participants, and then proceeded with two "mixed" practice blocks (i.e., alternating between the two tasks) of 48 and 72 trials, respectively. After practice, eight 72-trial mixed blocks were performed. Within-pairs RSIs were kept constant at $500 \mathrm{msec}$. (The longer within-pairs RSIs here, as compared with those in Experiment 1, were necessary to allow registration of vocal responses.)

\section{Results and Discussion}

Response time (RT) served as the primary dependent variable; values excluded from analysis were those faster than $200 \mathrm{msec}$, the longest .5\% of trials (above 2,500 msec and 1,772 $\mathrm{msec}$, respectively, for Experiments 1 and 2), error trials, trials immediately following errors, and trials in which the microphone was not properly triggered (in Experiment 2). Response repetition trials were also excluded in Experiment 2; response repetitions usually produce different effects for task repetitions (benefits) versus switches (costs). Specifically, stimulus-response repetitions on task-repetition transition trials may allow bypassing of the usual selection stage and thus render subtle effects more difficult to detect. ${ }^{2}$ However, response repetitions were not excluded in Experiment 1, given that repetition effects were very small in this experiment, and that, with only two different stimuli, this would have resulted in the loss of about half of the data.
Tables 1 and 2 show the complete RT and percent error results. Figure 2 presents the RT results as a function of task, position, and RSI for Experiments 1 and 2. As can be seen, there were large selection costs for both types of between-pairs transitions, but these costs were somewhat smaller for the task-repetition transitions than for the task-change transitions. As expected, the selection-cost asymmetry - that is, the interaction between the task and the position factor - was reliable overall in both experiments [Experiment 1, $F(1,35)=11.29, p<.01$; Experiment $2, F(1,29)=36.20, p<.001]$. This cost asymmetry was larger for task-change transitions in Experiment 2, as the interaction with transition type was highly reliable $[F(1,29)=14.65, p<.01]$; however, this interaction did not meet the reliability criterion in Experiment 1 $[F(1,35)=1.81, p=.19]$.

To test our predictions, it is critical to examine the asymmetry separately for each transition type: For taskchange transitions, the asymmetry was highly reliable [Experiment 1, $F(1,35)=13.28, p<.01$; Experiment 2, $F(1,29)=32.72, p<.001]$. However, as predicted, even for task-repetition transitions a reliable asymmetry was obtained [Experiment $1, F(1,35)=4.40, p<.05$; Experiment $2, F(1,29)=16.23, p<.001]$. The critical task $\times$ position interaction for task-repetition transitions was reliably modulated by RSI in Experiment $2[F(1,29)=8.83$, $p<.01]$, but not in Experiment $1[F(1,35)=.70, p=.41]$. When testing the task $\times$ position interaction separately for both the long and short RSIs (for task repetitions), the asymmetry proved reliable for the long RSI in Experiments 1 and $2[F(1,35)=4.15, p<.05$, and $F(1,29)=$ $21.9, p<.001$, respectively]. However, the asymmetry failed the reliability criterion for the short RSI (for task repetitions) in Experiment $1[F(1,35)=1.73, p=.20]$ and in Experiment $2[F(1,29)=1.88, p=.18]$.

As can be seen from Table 1, the error data from Experiment 1 generally followed the RT pattern, even though the critical asymmetry effects did not always meet the reliability criterion. Error effects from Experiment 2 were a bit more complex. In particular, a close look at the error pattern for the task-repetition transitions suggests some evidence for a speed-accuracy trade-off modulated by RSI. Numerically, the expected asymmetry pattern in terms of Position 1 versus

Table 1

Descriptive Statistics for Experiment 1

\begin{tabular}{|c|c|c|c|c|c|c|c|c|c|c|}
\hline \multirow[b]{3}{*}{ S-R Compatibility } & \multirow[b]{3}{*}{ RSI } & & \multicolumn{4}{|c|}{ Compatible } & \multicolumn{4}{|c|}{ Incompatible } \\
\hline & & & \multicolumn{2}{|c|}{ Position 1} & \multicolumn{2}{|c|}{ Position 2} & \multicolumn{2}{|c|}{ Position 1} & \multicolumn{2}{|c|}{ Position 2} \\
\hline & & & $M$ & $S D$ & $M$ & $S D$ & $M$ & $S D$ & $M$ & $S D$ \\
\hline \multirow[t]{3}{*}{ Task repetition } & Short & RT (msec) & 644 & 144 & 489 & 73 & 700 & 144 & 564 & 110 \\
\hline & & $\%$ Error & 0.68 & 1.51 & 0.39 & 1.29 & 0.83 & 1.54 & 1.65 & 2.43 \\
\hline & Long & $\begin{array}{l}\text { RT } \\
\% \text { Error }\end{array}$ & $\begin{array}{l}844 \\
1.00\end{array}$ & $\begin{array}{l}171 \\
1.99\end{array}$ & $\begin{array}{l}478 \\
0.34\end{array}$ & $\begin{array}{c}69 \\
0.86\end{array}$ & $\begin{array}{l}876 \\
0.70\end{array}$ & $\begin{array}{l}160 \\
1.58\end{array}$ & $\begin{array}{l}547 \\
0.56\end{array}$ & $\begin{array}{r}95 \\
1.22\end{array}$ \\
\hline \multirow[t]{2}{*}{ Task change } & Short & $\begin{array}{l}\text { RT } \\
\% \text { Error }\end{array}$ & $\begin{array}{l}816 \\
6.38\end{array}$ & $\begin{array}{l}200 \\
4.32\end{array}$ & $\begin{array}{l}473 \\
0.37\end{array}$ & $\begin{array}{c}70 \\
0.96\end{array}$ & $\begin{array}{l}813 \\
4.46\end{array}$ & $\begin{array}{l}164 \\
3.67\end{array}$ & $\begin{array}{l}526 \\
0.61\end{array}$ & $\begin{array}{r}87 \\
1.61\end{array}$ \\
\hline & Long & $\begin{array}{l}\text { RT } \\
\% \text { Error }\end{array}$ & $\begin{array}{l}886 \\
4.05\end{array}$ & $\begin{array}{l}186 \\
4.28\end{array}$ & $\begin{array}{l}464 \\
0.14\end{array}$ & $\begin{array}{c}80 \\
0.61\end{array}$ & $\begin{array}{l}904 \\
2.12\end{array}$ & $\begin{array}{l}164 \\
2.55\end{array}$ & $\begin{array}{l}520 \\
0.51\end{array}$ & $\begin{array}{r}84 \\
1.29\end{array}$ \\
\hline
\end{tabular}

Note-Short $=$ between-pairs response-to-stimulus interval (RSI) of $500 \mathrm{msec}$; Long $=$ between-pairs RSI of 5,000 msec. 
Table 2

Descriptive Statistics for Experiment 2

\begin{tabular}{|c|c|c|c|c|c|c|c|c|c|c|}
\hline \multirow[b]{3}{*}{ Stroop } & \multirow[b]{3}{*}{ RSI } & & \multicolumn{4}{|c|}{ Word } & \multicolumn{4}{|c|}{ Color } \\
\hline & & & \multicolumn{2}{|c|}{ Position 1} & \multicolumn{2}{|c|}{ Position 2} & \multicolumn{2}{|c|}{ Position 1} & \multicolumn{2}{|c|}{ Position 2} \\
\hline & & & $M$ & $S D$ & $M$ & $S D$ & $M$ & $S D$ & $M$ & $S D$ \\
\hline \multirow[t]{4}{*}{ Task repetition } & Short & RT (msec) & 704 & 76 & 597 & 102 & 887 & 102 & 795 & 118 \\
\hline & & $\%$ Error & 1.70 & 2.51 & 2.77 & 4.75 & 2.47 & 3.51 & 5.14 & 6.07 \\
\hline & Long & RT & 788 & 96 & 569 & 81 & 924 & 97 & 765 & 106 \\
\hline & & $\%$ Error & 1.28 & 2.47 & 3.28 & 4.83 & 2.89 & 4.60 & 2.80 & 4.59 \\
\hline \multirow[t]{4}{*}{ Task change } & Short & RT & 884 & 94 & 614 & 116 & 971 & 96 & 793 & 118 \\
\hline & & $\%$ Error & 6.02 & 6.41 & 2.19 & 3.82 & 2.85 & 4.09 & 4.69 & 6.22 \\
\hline & Long & RT & 898 & 113 & 578 & 99 & 1,002 & 106 & 788 & 107 \\
\hline & & \% Error & 4.46 & 4.97 & 3.21 & 4.86 & 3.07 & 4.62 & 2.41 & 4.13 \\
\hline
\end{tabular}

Note-Short $=$ between-pairs response-to-stimulus interval $(\mathrm{RSI})$ of $500 \mathrm{msec}$; Long $=$ between-pairs RSI of 5,000 msec.

Position 2 selection costs was found for the short RSI, but a reversed pattern (i.e., color-naming costs larger than wordnaming selection costs) was found for the long RSI. Neither of these effects was reliable, nor was the interaction of RSI, task, and position. Nevertheless, to ensure that the RT effects were not compromised by a trade-off with accuracy, we looked at the RT asymmetry effects in the critical condition (task-repetition, long RSI) for just those participants ( $n=$ 18) who showed the "standard" asymmetry pattern in their error scores and found that they did observe a reliable RT asymmetry in the correct direction $[F(1,17)=17.73, p<$ $.01]$. Thus, combined with the results from Experiment 1,

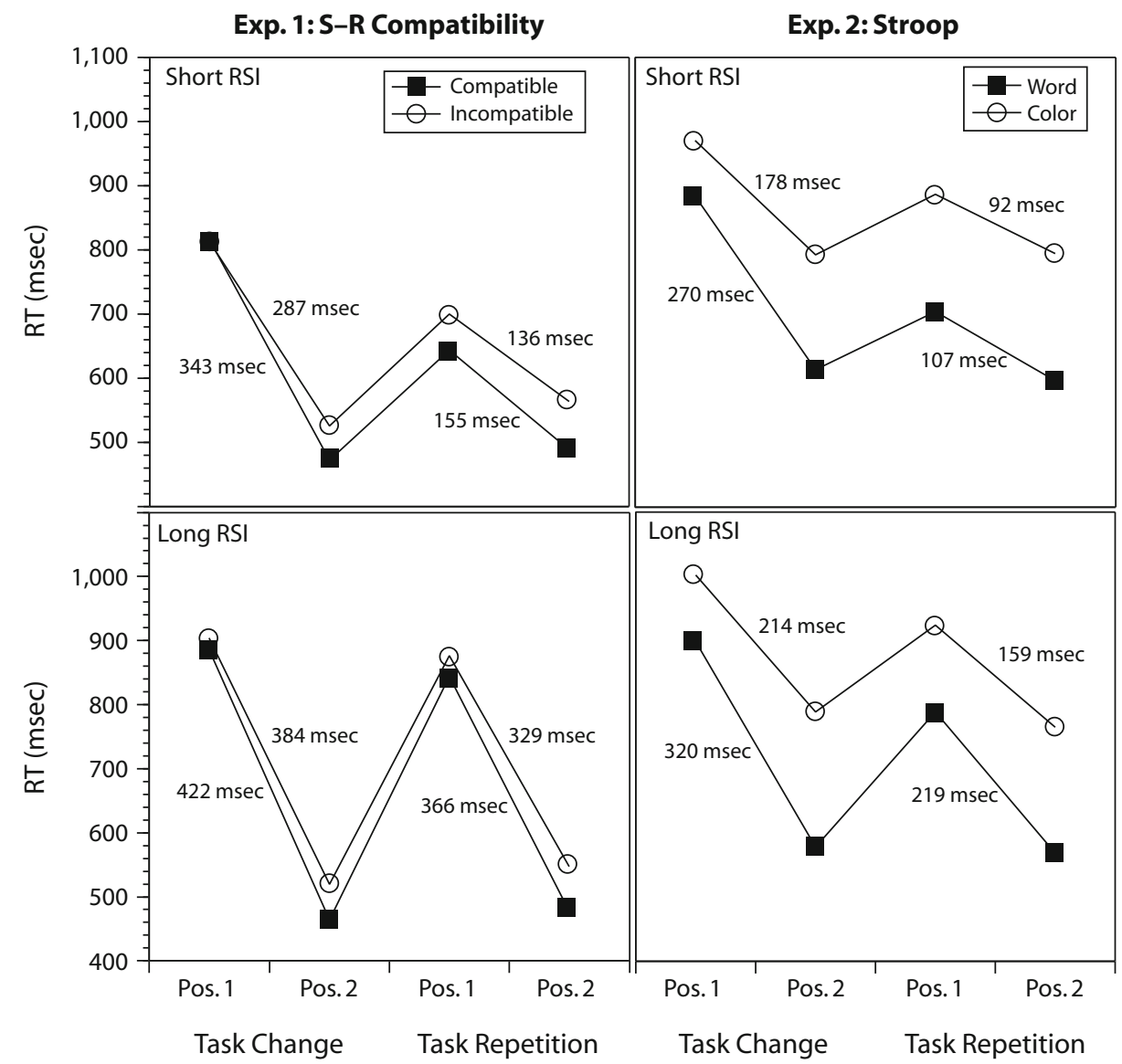

Figure 2. Mean response time (RT) shown as a function of task, position, task transition type, and RSI for Experiment 1 (left panel) and Experiment 2 (right panel). Short = between-pairs RSI of 500 msec; Long = between-pairs RSI of 5,000 msec. Values shown next to each line indicate "selection costs" (i.e., the difference between Position 1 and Position 2 within a trial pair) for the respective RSI $\times$ task $\times$ transition type condition. 
Table 3

Mean Percent Errors for Experiment 3A (Within Subjects) and Experiment 3B (Between Subjects)

\begin{tabular}{|c|c|c|c|c|c|c|c|c|c|}
\hline & \multirow[b]{3}{*}{ RSI } & \multicolumn{4}{|c|}{ Word } & \multicolumn{4}{|c|}{ Color } \\
\hline & & \multicolumn{2}{|c|}{ Position 1} & \multicolumn{2}{|c|}{ Position 2} & \multicolumn{2}{|c|}{ Position 1} & \multicolumn{2}{|c|}{ Position 2} \\
\hline & & $M$ & $\frac{S D}{S D}$ & $M$ & $\overline{S D}$ & $M$ & $\overline{S D}$ & $M$ & $S D$ \\
\hline \multirow[t]{2}{*}{ Within subjects } & Short & 0.00 & 0.00 & 0.00 & 0.00 & 1.98 & 1.68 & 1.87 & 2.23 \\
\hline & Long & 0.10 & 0.37 & 0.10 & 0.38 & 0.42 & 0.69 & 1.15 & 1.30 \\
\hline \multirow[t]{2}{*}{ Between subjects } & Short & 0.22 & 0.59 & 0.14 & 0.36 & 1.08 & 1.20 & 1.46 & 1.31 \\
\hline & Long & 0.00 & 0.00 & 0.07 & 0.25 & 0.51 & 0.88 & 1.45 & 0.99 \\
\hline
\end{tabular}

Note-Short $=$ between-pairs response-to-stimulus interval $(\mathrm{RSI})$ of $500 \mathrm{msec}$; Long $=$ between-pairs RSI of 5,000 msec.

where there was no indication of a speed-accuracy trade-off, we can be relatively confident that the RT asymmetry for task repetition is a real phenomenon.

\section{EXPERIMENTS 3A AND 3B}

\section{Method}

In Experiments 3A and 3B, we attempted to provide a still stronger test of the LTM-retrieval view of asymmetric costs. According to this account, the greater difficulty with selecting the dominant task arises from LTM interference. If this is correct, the effect of such LTM influences should be detectable beyond the type of rapid back-and-forth task-switching situations used in Experiments 1 and 2. Specifically, we predicted that dominant task RTs would be increased in single-task blocks when these were intermixed with nondominant single-task blocks. Again, this effect should be particularly strong for long RSIs where frequent occasions of a "loss of set" and subsequent acts of re-retrieving the task can be expected. Thus, in this context, the cost asymmetry should become apparent as a larger increase of RTs for the dominant than for the nondominant task as a function of an increase in RSI.

In Experiment 3B, we also contrasted dominant and nondominant single-task performance in a between-subjects design that was otherwise completely analogous to Experiment 3A. It is possible that the type of asymmetry in long RSI, task-repetition trials we have seen in Experiments 1 and 2 is an unspecific phenomenon that has nothing to do with competition between tasks. For example, people might habitually prepare for short RSIs, so that long RSIs produce an expectancy violation (e.g., Gottsdanker, 1979) to which, in turn, easy tasks might be more sensitive than difficult tasks. ${ }^{3}$ If the cost asymmetry is actually tied to competition between tasks, then eliminating such competition with the between-subjects design should lead to identical RSI effects for dominant and nondominant tasks.

Participants. Sixteen participants were included in the withinsubjects Experiment $3 \mathrm{~A}$, and 32 participants were included in the between-subjects Experiment 3B.

General Design. Experiment 3A was identical to Experiment 2, including all aspects of the stimuli, the display, and the RSI manipulation, except that participants only performed a single task (word naming or color naming) during each block. Participants alternated between 72-trial word- and color-naming blocks in an ABBAABBA sequence, counterbalanced across participants, by which task was presented first. Prior to actual testing, participants were given one 72-trial practice block each of color and word naming.

Experiment 3B was identical to Experiment 3A, except that the task condition was manipulated between subjects, such that a given participant only performed one of the tasks (color naming or word naming). Practice involved two blocks of 72 trials of a given task.

\section{Results}

The criteria used for excluding trials were identical to those for Experiment 2. Figure 3 shows RTs as a function of task, position, and RSI within a task pair for both the within-subjects (Experiment 3A) and the between-subjects (Experiment 3B) experiments. We had predicted that, when between-tasks competition is high (Experiment 3A)

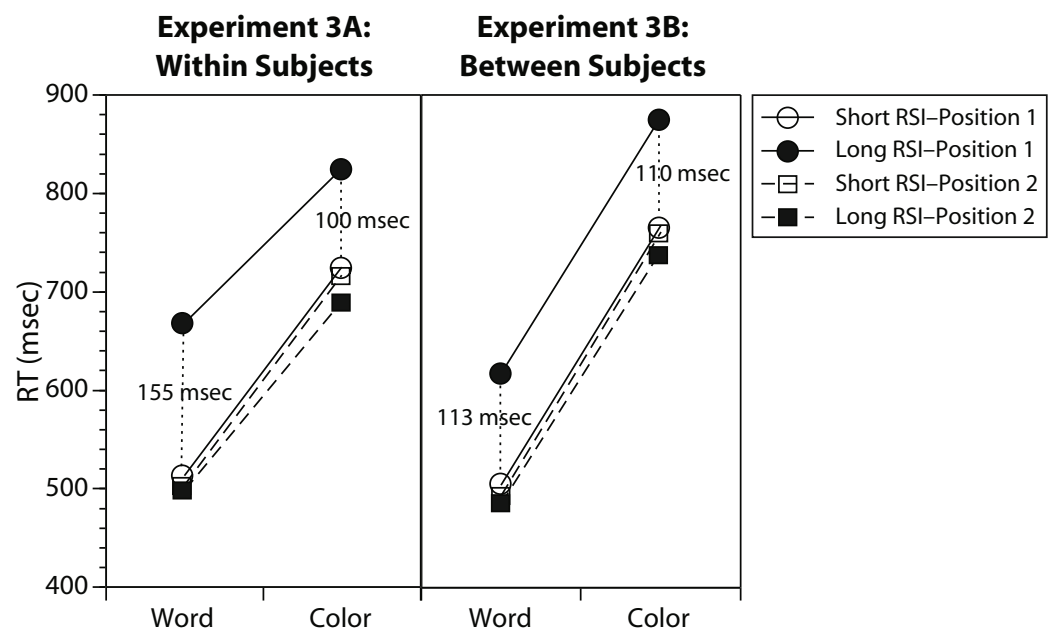

Figure 3. Mean response time (RT) for Experiment 3A (left panel) and Experiment 3B (right panel) as a function of RSI, position, and task. Short $=$ between-pairs RSI of 500 msec; Long = between-pairs RSI of 5,000 msec. 
and there is a high likelihood of losing the task set from working memory (i.e., Position 1 after long RSIs), RTs for the dominant word task should be prolonged in comparison with those for the color task. As is evident in the left panel of Figure 3, this was the case. The critical interaction of task, position, and RSI was highly reliable $[F(1,16)=$ $10.76, p<.01]$ for Experiment 3A. Specifically, the RT increase as a function of RSI was considerably larger for the word than for the color task on Position 1 trials $(M=$ $155 \mathrm{msec}, S D=72 \mathrm{msec}$, and $M=100 \mathrm{msec}, S D=$ $62 \mathrm{msec}$, respectively).

Experiment 3B matched Experiment $3 \mathrm{~A}$ in all respects, except that the task factor was manipulated between subjects, thus eliminating direct competition between the dominant and the nondominant tasks. Consistent with the assumption that the cost asymmetry is not an unspecific phenomenon, but is driven by competition between tasks, the RSI effect was highly similar for the two tasks here (Position 1 word = $113 \mathrm{msec}, S D=59 \mathrm{msec}$, Position 1 color $=109 \mathrm{msec}, S D=65$ ), and there was no hint of a reliable interaction of task, RSI, and position $[F(1,26)=$ $.04, p=.84] .{ }^{4}$ Overall, error rates were very low and did not counteract the critical pattern of RT results (see Table 3).

We also directly contrasted the asymmetry for the Position 1 selection costs (i.e., long RSI vs. short RSI) between Experiments $3 \mathrm{~A}$ and $3 \mathrm{~B}$, using a procedure for comparing effects from within- versus between-subjects designs (Altmann, 2002; Erlebacher, 1977). ${ }^{5}$ The asymmetry for the selection costs was reliably larger in the within- than in the between-subjects design $[F(1,20.58)=4.60, p<.05]$, confirming that between-tasks competition is, in fact, critical to the emergence of asymmetric costs.

\section{GENERAL DISCUSSION}

Taken together, the three experiments reported here provided clear evidence that a switch in task is a sufficient, but not a necessary, condition to obtain a selection cost asymmetry (see also Allport \& Wylie, 2000). A cost asymmetry was obtained both on trials in which the task changed and on trials in which the task repeated when there was a long delay between trials. We had hypothesized that long delays would increase the likelihood of losing the previous trial set and would therefore enforce retrieval of task-set information from LTM, just as would otherwise occur during a switch in task (e.g., Mayr \& Kliegl, 2000). The idea that cost asymmetries result from LTM influences is particularly strengthened by the fact that an asymmetry was obtained in Experiment $3 \mathrm{~A}$, even though the two tasks were separated into distinct blocks, thus ruling out any role of short-term priming effects.

The present demonstration of a selection cost asymmetry, even in the absence of an actual task switch, is theoretically important because it cannot be explained by carryover accounts of task-switch costs (see also Allport $\&$ Wylie, 2000). By these accounts, an actual switch in tasks is a necessary condition for an asymmetry. We do not, however, want to claim that such models could not be amended to produce the observed asymmetries. For example, Gilbert and Shallice (2002) speculated (in a somewhat different context) about the consequences of allowing long-term strengthening of associations between stimulus-response and task-set nodes, rather than resetting them from trial to trial. However, the required amendments would clearly move the continuous-activation accounts closer to the LTM-retrieval account proposed here.

So far, we have alluded to the LTM-retrieval view of task selection only in a very general manner. How exactly would this account explain the cost asymmetry? One possibility we currently favor is the following: Assume that each task-selection instance, whether after a switch or after a loss of the currently relevant task set over time, requires retrieval of the relevant task information from LTM. In addition, each trial also provides a learning opportunity that, with some probability, adds traces encoding specific features about that selection instance to LTM (Logan, 1988, 1990; Mayr \& Bryck, 2005). Past work has already shown that automatic retrieval of such memory traces provides at least a partial explanation for switch costs (Waszak, Hommel, \& Allport, 2003). However, an additional assumption is necessary to explain the selection-cost asymmetry: The probability of encoding a memory trace needs to increase as a function of attentional control exerted during the selection instance. Assuming that more attentional control is necessary for nondominant than for dominant tasks, more traces representing nondominant task instances would be laid down in LTM than for dominant task instances. As a result, during attempts to retrieve task-set information from LTM (e.g., on switch or restart trials), there is a greater danger of interference through nondominant task traces than through dominant task traces.

There is some evidence in the memory literature that encoding efficiency is a direct function of how much attention is devoted to the to-be-encoded information (see, e.g., Craik, Govoni, Naveh-Benjamin, \& Anderson, 1996). In future work, it will be important to provide additional, more direct evidence for the critical claim that greater interference during encoding, such as via a dominant competitor task, actually leads to the encoding of more memory traces for the nondominant than for the dominant task.

Irrespective of how this particular theoretical account of asymmetric costs will hold up in future work, the critical finding of this study is that we could confirm and extend reports by Allport and Wylie (2000) that switch transitions are not a necessary condition for asymmetric costs. As a result, the intuitively appealing idea that switch costs and switch cost asymmetries arise from trial-to-trial carryover effects may have to be reconsidered.

\section{AUTHOR NOTE}

We thank Lara Barnes and Rebekah Napack for running participants in these experiments. Correspondence concerning this article should be addressed to R. L. Bryck, Department of Psychology, 1227 University of Oregon, Eugene, OR 97403 (e-mail: rbryck@uoregon.edu).

\section{REFERENCES}

Allport, A., Styles, E. A., \& Hsieh, S. (1994). Shifting intentional set: Exploring the dynamic control of tasks. In C. Umiltà \& M. Mos- 
covitch (Eds.), Attention and performance XV: Conscious and nonconscious information processing (pp. 421-452). Cambridge, MA: MIT Press.

Allport, A., \& Wylie, G. (2000). Task-switching, stimulus-response bindings, and negative priming. In S. Monsell \& J. S. Driver (Eds.) Attention and performance XVIII: Control of cognitive processes (pp. 35-70). Cambridge, MA: MIT Press.

Altmann, E. M. (2002). Functional decay of memory for tasks. Psychological Research, 66, 287-297.

Craik, F. I. M., Govoni, R., Naveh-Benjamin, M., \& Anderson, N. D. (1996). The effects of divided attention on encoding and retrieval processes in human memory. Journal of Experimental Psychology: General, 125, 159-180.

ERLEBACHER, A. (1977). Design and analysis of experiments contrasting the within- and between-subjects manipulation of the independent variable. Psychological Bulletin, 84, 212-219.

Gilbert, S. J., \& Shallice, T. (2002). Task switching: A PDP model. Cognitive Psychology, 44, 297-337.

Gopher, D., Armony, L., \& Greenshpan, Y. (2000). Switching tasks and attention policies. Journal of Experimental Psychology: General, 129, 308-339.

GotTSDANKer, R. (1979). A psychological refractory period or an unprepared period? Journal of Experimental Psychology: Human Perception \& Performance, 5, 208-215.

Hazy, T. E., FranK, M. J., \& O'ReILly, R. C. (2006). Banishing the homunculus: Making working memory work. Neuroscience, 139, 105-118.

LiEN, M.-C., \& RuthrufF, E. (2004). Task switching in a hierarchical task structure: Evidence for the fragility of the task repetition benefit. Journal of Experimental Psychology: Learning, Memory, \& Cognition, 30, 697-713.

Logan, G. D. (1988). Toward an instance theory of automatization. Psychological Review, 95, 492-527.

Logan, G. D. (1990). Repetition priming and automaticity: Common underlying mechanisms? Cognitive Psychology, 22, 1-35.

MAYR, U., \& BRYCK, R. L. (2005). Sticky rules: Integration between abstract rules and specific actions. Journal of Experimental Psychology: Learning, Memory, \& Cognition, 31, 337-350.

Mayr, U., \& Kliegl, R. (2000). Task-set switching and long-term memory retrieval. Journal of Experimental Psychology: Learning, Memory, \& Cognition, 26, 1124-1140.
MAYR, U., \& KLIEGL, R. (2003). Differential effects of cue changes and task changes on task-set selection costs. Journal of Experimental Psychology: Learning, Memory, \& Cognition, 29, 362-372.

Rogers, R. D., \& Monsell, S. (1995). Costs of a predictable switch between simple cognitive tasks. Journal of Experimental Psychology: General, 124, 207-231.

Waszak, F., Hommel, B., \& Allport, A. (2003). Task-switching and long-term priming: Role of episodic stimulus-task bindings in taskshift costs. Cognitive Psychology, 46, 361-413.

YeUnG, N., \& MonselL, S. (2003). Switching between tasks of unequal familiarity: The role of stimulus-attribute and response-set selection. Journal of Experimental Psychology: Human Perception \& Performance, 29, 455-469.

\section{NOTES}

1. This is actually not a fully complete account of how the asymmetry arises in this model. The additional aspect to consider is that for the color task, but not for the word task, a relatively large task-demand input is required even on no-switch trials. Thus, there is a very large difference for word-demand units between no-switch and switch trials, whereas the relative difference for color-demand units is much smaller.

2. When not eliminating response repetitions, the critical pattern of results is weakened, but remains reliable.

3. We thank Gordon Logan for suggesting this possibility.

4. It should be noted that this analysis includes the full set of data for Experiment 3B. It could be argued that it is more appropriate to analyze only the first half of the data for this experiment, given that each participant performed the same number of total trials as participants in Experiment $3 \mathrm{~A}$ did, thereby doubling the number of trials for each task. However, when analyzed this way, the overall pattern of results stays the same. The critical interaction of the task, RSI, and position is again far from reliable $[F(1,26)=.29, p=.60]$.

5. We thank Erik Altmann for pointing us to this procedure.

(Manuscript received November 8, 2006; revision accepted for publication July 21, 2007.) 